\title{
Strongly extreme points of Orlicz function spaces equipped with $\Phi$-Amemiya norm
}

\section{Lili An ${ }^{1 *}$ and Yunan Cui ${ }^{1}$ (B)}

"Correspondence:

anlili_935@163.com

1 Harbin University of Science and

Technology, Harbin, China

\section{Springer}

\begin{abstract}
In this paper, the criterion that points of Orlicz function spaces equipped with $\Phi$-Amemiya norm generated by an Orlicz function are strongly extreme is given. As a corollary, the sufficient and necessary conditions of midpoint local uniform rotundity of Orlicz function spaces equipped with $\Phi$-Amemiya norm are obtained.
\end{abstract}

MSC: Primary 39B82; secondary 44B20; 46C05

Keywords: Orlicz function spaces; $\Phi$-Amemiya norm; Strongly extreme points; Midpoint local uniform rotundity

\section{Introduction}

An extreme point plays a crucial role in functional analysis, convex analysis, and optimization. In fact, any compact convex set is the convex hull of its extreme point set, the result is called Krein-Milman theorem. The notion of a dentable subset of a Banach space was introduced by Rieffel in conjunction with a Radon-Nikodym theorem for Banach spacevalued measures. Subsequent work by Maynard and Davis and Phelos has shown those Banach spaces in which Rieffel's Radon-Nikodym theorem is valid and every bounded closed convex set is dentable. This has been a significant breakthrough in studying the nature of Radon-Nikodym as a geometric property. In 1988, Bor-Luh Lin, Pei-Kee Lin, and Troyanski described the characteristic of denting points (see $[1,2]$ ) and obtained that there is a close relationship between denting points and strongly extreme points. It is easy to see that every denting point of Banach space $X$ is a strongly extreme point (see [3]) of $X$, and it is known that every strongly extreme point of $X$ is a $w^{*}$ extreme point of $X$. Orlicz space is an important class of Banach space, it was introduced by the famous Polish mathematician Wladyslaw Orlicz in 1932. The theory of Orlicz space has been greatly developed because of its important theoretical properties and application value. Up to now, the criterion that an element in the unit sphere of Orlicz spaces equipped with the Orlicz norm, the Luxemburg norm, and the $p$-Amemiya norm is a strongly extreme point has been obtained (see [4-6]). In this paper, we introduce a new norm, namely $\Phi$-Amemiya norm, whose calculation formula is given as follows: $\|x\|_{\Phi, \Phi_{1}}=\inf _{k>0}\left\{\frac{1}{k}\left(1+\Phi\left(I_{\Phi_{1}}(k x)\right)\right)\right\}$. When we take some special functions, the previous norms are special cases of this new norm. This new norm also has wider applicability than before. We give the criterion that an element in the

(c) The Author(s) 2020. This article is licensed under a Creative Commons Attribution 4.0 International License, which permits use sharing, adaptation, distribution and reproduction in any medium or format, as long as you give appropriate credit to the original author(s) and the source, provide a link to the Creative Commons licence, and indicate if changes were made. The images or other third party material in this article are included in the article's Creative Commons licence, unless indicated otherwise in a credit line to the material. If material is not included in the article's Creative Commons licence and your intended use is not permitted by statutory regulation or exceeds the permitted use, you will need to obtain permission directly from the copyright holder. To view a copy of this licence, visit http://creativecommons.org/licenses/by/4.0/. 
unit sphere of Orlicz spaces equipped with $\Phi$-Amemiya norm is a strongly extreme point. Incidentally, the sufficient and necessary conditions of midpoint local uniform rotundity of Orlicz function spaces equipped with $\Phi$-Amemiya norm are obtained.

\section{Preliminaries}

Let $[X,\|\cdot\|]$ be a Banach space. $S(X)$ and $B(X)$ denote the unit sphere and the unit ball of $X$, respectively. $X^{*}$ is said to be the dual space of $X$.

Definition 2.1 A mapping $\Phi: R \rightarrow[0, \infty)$ is called an Orlicz function: if $\Phi$ is even, continuous, convex and $\Phi(u)=0$ if and only if $u=0$. If $\Phi$ also satisfies $\lim _{u \rightarrow 0} \frac{\Phi(u)}{u}=0$ and $\lim _{u \rightarrow \infty} \frac{\Phi(u)}{u}=\infty$, then $\Phi$ is called an $N$-function.

Definition 2.2 The function $\Psi$ defined by the formula $\Psi(u)=\sup \{|u| v-\Phi(v): v \geq 0\}$ is called complementary function of $\Phi$ in the sense of Young.

Definition 2.3 Let $(G, \Sigma, \mu)$ be a nonatomic finite measure space. Let $L^{0}$ denote the whole of the measurable real function on $G$. We define the modular $I_{\Phi}: L^{0} \rightarrow R^{+}=[0,+\infty]$ as follows:

$$
I_{\Phi}(x)=\int_{G} \Phi(x(t)) d t
$$

it is called the modular (see [7]) of $x$.

Definition 2.4 The Orlicz function space (see [8]) $L_{\Phi}$ generated by an Orlicz function is defined by the formula $L_{\Phi}=\left\{x \in L^{0}: I_{\Phi}(k x)<+\infty\right.$ for some $\left.k>0\right\}$.

Those spaces that are equipped with the Orlicz norm (Amemiya norm) (see [9])

$$
\|x\|_{\Phi}^{0}=\inf _{k>0} \frac{1}{k}\left(1+I_{\Phi}(k x)\right),
$$

or equipped with the Luxemburg norm

$$
\|x\|_{\Phi}=\inf \left\{k>0: I_{\Phi}\left(\frac{x}{k}\right) \leq 1\right\}
$$

or equipped with the $p$-Amemiya norm $(1 \leq p<+\infty)$ (see $[10,11])$

$$
\|x\|_{\Phi, p}=\inf _{k>0} \frac{1}{k}\left(1+I_{\Phi}^{p}(k x)\right)^{\frac{1}{p}},
$$

are Banach spaces, abbreviated as

$$
L_{\Phi}^{0}=\left[L_{\Phi},\|\cdot\|_{\Phi}^{0}\right] ; \quad L_{\Phi}=\left[L_{\Phi},\|\cdot\|_{\Phi}\right] ; \quad L_{\Phi, p}=\left[L_{\Phi},\|\cdot\|_{\Phi, p}\right] .
$$

Definition 2.5 We say that Orlicz function $\Phi$ satisfies the $\Delta_{2}$ condition if there exist $k>2$ and $u_{0} \geq 0$ such that the inequality

$$
\Phi(2 u) \leq k \Phi(u)
$$

holds for $|u| \geq u_{0}$. 
Definition 2.6 If, for every $y, z \in R$ and $y \neq z$ with $\frac{y+z}{2}=x$, we have $\Phi(x)<\frac{\Phi(y)+\Phi(z)}{2}$, then $x$ is called a strictly convex point of $\Phi$. The set of all strictly convex points of $\Phi$ will be denoted by $S_{\Phi}$.

For any Orlicz functions $\Phi$ and $\Phi_{1}$, we put $L_{\Phi, \Phi_{1}}=\left\{x \in L^{0}: \Phi\left(I_{\Phi_{1}}(k x)\right)<+\infty\right.$ for some $k>0\}$. The calculation formula

$$
\|x\|_{\Phi, \Phi_{1}}=\inf _{k>0} \frac{1}{k}\left(1+\Phi\left(I_{\Phi_{1}}(k x)\right)\right)
$$

is called $\Phi$-Amemiya norm.

\section{Remark}

If we take $\Phi(u)=\max \{0, u-1\}$, then $\|x\|_{\Phi, \Phi_{1}}$ is the Luxemburg norm $\|\cdot\|_{\Phi}$;

If we take $\Phi(u)=u$, then $\|x\|_{\Phi, \Phi_{1}}$ is the Orlicz norm $\|\cdot\|_{\Phi}^{0}$;

If we take $\max \{0, u-1\} \leq \Phi(u) \leq u$, then $\|x\|_{\Phi, \Phi_{1}}$ is the $s$-norm $\|\cdot\|_{\Phi}^{s}$;

If we take $\Phi(u) \geq|u|$, then $\|x\|_{\Phi, \Phi_{1}} \geq\|\cdot\|_{\Phi}^{0}$.

An important question is the attainability of the "inf" in $\|x\|_{\Phi, \Phi_{1}}=\inf _{k>0} \frac{1}{k}(1+$ $\left.\Phi\left(I_{\Phi_{1}}(k x)\right)\right)$. For any $x \in L_{\Phi, \Phi_{1}}, x \neq 0$, we define

$$
K(x)=\left\{k>0:\|x\|_{\Phi, \Phi_{1}}=\frac{1}{k}\left(1+\Phi\left(I_{\Phi_{1}}(k x)\right)\right)\right\} .
$$

We will prove that if $\lim _{u \rightarrow \infty} \frac{\Phi_{1}(u)}{u}=+\infty$, then $K(x) \neq \phi$.

Proof Put $F(k)=\frac{1}{k}\left(1+\Phi\left(\int_{G} \Phi_{1}(k x(t)) d t\right)\right)$ and $\theta(x)=\inf \left\{k>0, I_{\Phi_{1}}\left(\frac{x}{k}\right)<\infty\right\}$.

Then there exists $d>0$ such that $\mu(\{t \in G:|x(t)|>d\})>0$ and $F(k)$ is continuous on $(0, \theta(x))$. So $\lim _{k \rightarrow 0^{+}} F(k)=+\infty$.

Suppose that $\theta(x)=+\infty$. Then

$$
\begin{aligned}
\lim _{k \rightarrow+\infty} F(k) & =\lim _{k \rightarrow+\infty} \frac{\Phi\left(\int_{G} \Phi_{1}(k x(t)) d t\right)}{k} \\
& \geq \lim _{k \rightarrow+\infty} \Phi\left(\frac{\int_{G} \Phi_{1}(k x(t)) d t}{k}\right) \\
& \geq \lim _{k \rightarrow+\infty} \Phi\left(\frac{\int_{\{t \in G:|x(t)|>d\}} \Phi_{1}(k x(t)) d t}{k}\right) \\
& \geq d \lim _{k \rightarrow+\infty} \Phi\left(\frac{\Phi_{1}(k d) \cdot \mu(\{t \in G:|x(t)|>d\})}{k d}\right) \\
& =+\infty .
\end{aligned}
$$

Since $F(k)$ is a continuous function, then there exists $k_{0} \in(0, \theta(x))$ such that $F(k) \geq F\left(k_{0}\right)$.

Suppose that $\theta(x)<+\infty$. If $I_{\Phi_{1}}(\theta(x) x(t))=+\infty$, we have

$$
\lim _{k \rightarrow \theta(x)-0} F\left(k=\frac{1}{\theta(x)}\left(1+\Phi\left(\int_{G} \Phi_{1}(\theta(x) x(t)) d t\right)\right)=+\infty .\right.
$$


If $I_{\Phi_{1}}(\theta(x) x(t))<+\infty$, we have

$$
\lim _{k \rightarrow \theta(x)-0} F(k)=\frac{1}{\theta(x)}\left(1+\Phi\left(\int_{G} \Phi_{1}(\theta(x) x(t)) d t\right)\right)<+\infty .
$$

Since $F(k)$ is a continuous function, there exists $k_{0} \in(0, \theta(x)]$ such that $F(k) \geq F\left(k_{0}\right)$.

Thus $K(x) \neq \phi$.

Definition 2.7 A point $x \in S(X)$ is said to be an extreme point (see [12]) of $B(X)$ if, for any $y, z \in S(X)$ and $x=\frac{y+z}{2}$, we have $y=z$. The set of all extreme points of the unit ball $B(X)$ will be denoted by $\operatorname{Ext} B(X), X$ is said to be strictly convex (see [13]) if and only if $\operatorname{ExtB}(X)=S(X)$.

Definition 2.8 A point $x \in S(X)$ is called a strongly extreme point (see [14-16]) of $B(X)$ if, for any $\left\{x_{n}\right\} \subseteq X,\left\{y_{n}\right\} \subseteq X, \lim _{n \rightarrow \infty}\left\|x_{n}\right\|=\lim _{n \rightarrow \infty}\left\|y_{n}\right\|=1$, and $\frac{x_{n}+y_{n}}{2}=x$, we have $\lim _{n \rightarrow \infty}\left\|x_{n}-y_{n}\right\|=0$.

Definition 2.9 Banach space $X$ is called middle point local uniform convex (see $[17,18]$ ) if and only if each point on $S(X)$ is a strongly extreme point.

Lemma 2.10 (EropoB theorem) Let $\left\{f_{n}\right\}_{n=1}^{\infty}$ be a measurable function and $\left|f_{n}(x)\right|<\infty$ a.e. $x \in E$ with $m(E)<+\infty$. If $f_{n}(x) \rightarrow f(x)$ a.e. $x \in E$, then for any $\delta>0$ there exists $E_{0} \subset E$ such that $m\left(E_{0}\right)<\delta$ and $f_{n}(x) \rightarrow f(x)$ uniformly in $x \in E \backslash E_{0}$.

Lemma 2.11 Assume $\Phi \in \triangle_{2}$ (see [19]). Then, for any $L>0$ and $\varepsilon>0$, there exists $\delta>0$ such that

$$
\left|I_{\Phi}(u+v)-I_{\Phi}(v)\right|<\varepsilon,
$$

whenever $I_{\Phi}(u) \leq L, I_{\Phi}(v) \leq \delta$.

Lemma 2.12 Let $\Phi \in \triangle_{2}$. If $I_{\Phi}\left(x_{n}\right) \rightarrow I_{\Phi}(x), x_{n} \stackrel{\mu}{\rightarrow} x$, then $\left\|x_{n}-x\right\|_{\Phi} \rightarrow 0$ (see [19]).

\section{Main results}

Theorem 3.1 Let $\Phi_{1}$ be an N-function. Then $x_{0} \in S\left(L_{\Phi, \Phi_{1}}\right)$ is a strongly extreme point of $B\left(L_{\Phi, \Phi_{1}}\right)$ if and only if $\Phi_{1} \in \Delta_{2}$ and $k_{0} x_{0}(t) \in S_{\Phi_{1}}$, where $k_{0} \in K\left(x_{0}\right)$.

Proof Necessity. Suppose that $\mu\left(\left\{t \in G: k_{0} x_{0}(t) \notin S_{\Phi_{1}}\right\}\right)>0$ for some $k_{0} \in K\left(x_{0}\right)$. There exists an interval $(a, b)$ such that $\mu\left(\left\{t \in G: \frac{a}{k_{0}}+\varepsilon<x_{0}(t)<\frac{b}{k_{0}}-\varepsilon\right\}\right)>0(\varepsilon>0)$ and $\Phi_{1}$ is affine on $(a, b)$, i.e., $\Phi_{1}(x)=p x+q$. Divide $\left\{t \in G: \frac{a}{k_{0}}+\varepsilon<x_{0}(t)<\frac{b}{k_{0}}-\varepsilon\right\}$ into two sets $E$ and $F$ with $E \cap F=\emptyset$ and $\mu(E)=\mu(F)$. Define

$$
\begin{aligned}
& y(t)= \begin{cases}x_{0}(t), & t \in G \backslash(E \cup F), \\
x_{0}(t)-\varepsilon, & t \in E, \\
x_{0}(t)+\varepsilon, & t \in F,\end{cases} \\
& z(t)= \begin{cases}x_{0}(t), & t \in G \backslash(E \cup F), \\
x_{0}(t)+\varepsilon, & t \in E, \\
x_{0}(t)-\varepsilon, & t \in F .\end{cases}
\end{aligned}
$$


Then $x_{0}=\frac{y+z}{2}, y \neq z$ and

$$
\begin{aligned}
I_{\Phi_{1}}\left(k_{0} y\right)= & \int_{E \cup F} \Phi_{1}\left(k_{0} y(t)\right) d t+\int_{G \backslash E \cup F} \Phi_{1}\left(k_{0} y(t)\right) d t \\
= & \int_{E}\left(p\left(k_{0}\left(x_{0}(t)-\varepsilon\right)\right)+q\right) d t+\int_{F}\left(p\left(k_{0}\left(x_{0}(t)+\varepsilon\right)\right)+q\right) d t \\
& +\int_{G \backslash E \cup F} \Phi_{1}\left(k_{0} x_{0}(t)\right) d t \\
= & \int_{E \cup F}\left(p k_{0} x_{0}(t)+q\right) d t+\int_{G \backslash E \cup F} \Phi_{1}\left(k_{0} x_{0}(t)\right) d t \\
= & \int_{E \cup F} \Phi_{1}\left(k_{0} x_{0}(t)\right) d t+\int_{G \backslash E \cup F} \Phi_{1}\left(k_{0} x_{0}(t)\right) d t \\
= & I_{\Phi_{1}}\left(k_{0} x_{0}\right) .
\end{aligned}
$$

Thus $\|y\|_{\Phi, \Phi_{1}} \leq \frac{1}{k_{0}}\left(1+\Phi\left(I_{\Phi_{1}}\left(k_{0} y\right)\right)\right)=\frac{1}{k_{0}}\left(1+\Phi\left(I_{\Phi_{1}}\left(k_{0} x_{0}\right)\right)\right)=\left\|x_{0}\right\|_{\Phi, \Phi_{1}}=1$. In the same way, we can get $\|z\|_{\Phi, \Phi_{1}} \leq 1$. This contradicts the fact that $x_{0}$ is an extreme point of $S\left(L_{\Phi, \Phi_{1}}\right)$.

In order to complete this proof, we need to prove that if $\Phi_{1} \notin \Delta_{2}$, there is not a strongly extreme point on the unit sphere of $L_{\Phi, \Phi_{1}}$. If $x_{0} \in S\left(L_{\Phi, \Phi_{1}}\right)$, then there exists $d>0$ such that $\mu\left(\left\{t \in G:\left|x_{0}(t)\right| \leq d\right\}\right)>0$. Suppose $\Phi_{1} \notin \Delta_{2}$, then there exists $u_{n}>0, u_{n} \uparrow \infty$ such that $\Phi_{1}\left(2 u_{n}\right)>2^{n} \Phi_{1}\left(u_{n}\right)(n=1,2, \ldots)$. Without loss of generality, we can assume that $\frac{1}{\Phi_{1}\left(u_{1}\right)}<$ $\mu(\{t \in G:|x(t)| \leq d\})$. Take $\left\{G_{n}\right\} \subset\{t \in G:|x(t)|<d\}$ with $G_{m} \cap G_{n}=\emptyset$ for any $m \neq n$, satisfying

$$
\mu\left(G_{n}\right)=\frac{1}{2^{n} \Phi_{1}\left(u_{n}\right)} \quad(n=1,2, \ldots) .
$$

Define

$$
\begin{aligned}
& x_{n}(t)= \begin{cases}x_{0}(t), & t \in G \backslash G_{n}, \\
x_{0}(t)+\frac{u_{n}}{k_{0}}, & t \in G_{n},\end{cases} \\
& y_{n}(t)= \begin{cases}x_{0}(t), & t \in G \backslash G_{n}, \\
x_{0}(t)-\frac{u_{n}}{k_{0}}, & t \in G_{n} .\end{cases}
\end{aligned}
$$

Then $x_{0}=\frac{x_{n}+y_{n}}{2}$ for each $n \in N$.

Put

$$
x_{n}(t)=x_{n}^{\prime}(t)+x_{n}^{\prime \prime}(t)
$$

where $x_{n}^{\prime}(t)=x_{0} \chi_{G \backslash G_{n}}(t)+\frac{u_{n}}{k_{0}} \chi_{G_{n}}(t), x_{n}^{\prime \prime}(t)=x_{0} \chi_{G_{n}}(t)$.

Since $\left\|x_{n}^{\prime \prime}\right\|_{\Phi, \Phi_{1}}=\left\|x_{0} \chi_{G_{n}}\right\|_{\Phi, \Phi_{1}} \leq d\left\|_{G_{n}}\right\|_{\Phi, \Phi_{1}} \rightarrow 0(n \rightarrow \infty)$, we have the inequality $\left\|x_{n}^{\prime}\right\|_{\Phi, \Phi_{1}} \geq\left\|x_{0} \chi_{G \backslash G_{n}}\right\|_{\Phi, \Phi_{1}} \geq\left\|x_{0}\right\|_{\Phi, \Phi_{1}}-\left\|x_{0} \chi_{G_{n}}\right\|_{\Phi, \Phi_{1}}$ holds. Therefore $\lim _{\bar{n} \rightarrow \infty}\left\|x_{n}^{\prime}\right\|_{\Phi, \Phi_{1}} \geq$ $\left\|x_{0}\right\|_{\Phi, \Phi_{1}}=1$.

By the definition of $\Phi$-Amemiya norm, we deduce that

$$
\left\|x_{n}^{\prime}\right\|_{\Phi, \Phi_{1}}=\inf _{k>0} \frac{1}{k}\left(1+\Phi\left(I_{\Phi_{1}}\left(k x_{n}^{\prime}\right)\right)\right)
$$




$$
\begin{aligned}
& \leq \frac{1}{k_{0}}\left(1+\Phi\left(I_{\Phi_{1}}\left(k_{0} x_{n}^{\prime}\right)\right)\right) \\
& \leq \frac{1}{k_{0}}+\frac{1}{k_{0}} \Phi\left(\int_{G} \Phi_{1}\left(k_{0}\left(x_{0} \chi_{G \backslash G_{n}}(t)+\frac{u_{n}}{k_{0}} \chi_{G_{n}}(t)\right)\right) d t\right) \\
& \leq \frac{1}{k_{0}}+\frac{1}{k_{0}} \Phi\left(\int_{G \backslash G_{n}} \Phi_{1}\left(k_{0} x_{0} \chi_{G \backslash G_{n}}(t)\right) d t+\int_{G_{n}} \Phi_{1}\left(u_{n} \chi_{G_{n}}(t)\right) d t\right) \\
& \leq \frac{1}{k_{0}}+\frac{1}{k_{0}} \Phi\left(I_{\Phi_{1}}\left(k_{0} x_{0}\right)+\Phi_{1}\left(u_{n}\right) \mu\left(G_{n}\right)\right) \\
& =\frac{1}{k_{0}}\left(1+\Phi\left(I_{\Phi_{1}}\left(k_{0} x_{0}\right)+\frac{1}{2^{n}}\right)\right) .
\end{aligned}
$$

Then

$$
\varlimsup_{n \rightarrow \infty}\left\|x_{n}^{\prime}\right\|_{\Phi, \Phi_{1}} \leq\left\|x_{0}\right\|_{\Phi, \Phi_{1}}=1
$$

Hence

$$
\lim _{n \rightarrow \infty}\left\|x_{n}\right\|_{\Phi, \Phi_{1}}=1
$$

In the same way, we have

$$
\lim _{n \rightarrow \infty}\left\|y_{n}\right\|_{\Phi, \Phi_{1}}=1
$$

But

$$
\begin{aligned}
I_{\Phi_{1}}\left(k_{0}\left(x_{n}-y_{n}\right)\right) & =\int_{G_{n}} \Phi_{1}\left(k_{0} \frac{2 u_{n}(t)}{k_{0}}\right) d t \\
& =\Phi_{1}\left(2 u_{n}\right) \mu\left(G_{n}\right) \geq 1 \quad(n=1,2, \ldots) .
\end{aligned}
$$

Therefore

$$
\left\|x_{n}-y_{n}\right\|_{\Phi, \Phi_{1}}=\frac{1}{k_{0}}\left\|2 u_{n} \chi_{G_{n}}\right\|_{\Phi, \Phi_{1}} \geq \frac{1}{k_{0}}\left\|2 u_{n} \chi_{G_{n}}\right\|_{\Phi_{1}} \geq \frac{1}{k_{0}}
$$

a contradiction.

Sufficiency. Let $\Phi_{1} \in \triangle_{2}$ and $x_{0} \in S\left(L_{\Phi, \Phi_{1}}\right)$ with $k_{0} x_{0}(t) \in S_{\Phi_{1}}$ for $k_{0} \in K\left(x_{0}\right)$. For any $x_{n}, y_{n} \in L_{\Phi, \Phi_{1}}$ such that

$$
\begin{aligned}
& \lim _{n \rightarrow \infty}\left\|x_{n}\right\|_{\Phi, \Phi_{1}}=1, \\
& \lim _{n \rightarrow \infty}\left\|y_{n}\right\|_{\Phi, \Phi_{1}}=1, \\
& x_{n}+y_{n}=2 x_{0}
\end{aligned}
$$

for each $n \in N$.

Take sequences of positive numbers $\left\{k_{n}\right\}$ and $\left\{h_{n}\right\}$ such that

$$
\begin{aligned}
\left\|x_{n}\right\|_{\Phi, \Phi_{1}} & \geq \frac{1}{k_{n}}\left(1+\Phi\left(I_{\Phi_{1}}\left(k_{n} x_{n}\right)\right)\right)-\frac{1}{n}, \\
\left\|y_{n}\right\|_{\Phi, \Phi_{1}} & \geq \frac{1}{h_{n}}\left(1+\Phi\left(I_{\Phi_{1}}\left(h_{n} y_{n}\right)\right)\right)-\frac{1}{n} .
\end{aligned}
$$


Define

$$
\begin{aligned}
& \tilde{x_{n}}(t)=\frac{x_{n}+x_{0}}{2}, \\
& \tilde{y_{n}}(t)=\frac{y_{n}+x_{0}}{2} .
\end{aligned}
$$

Then

$$
\widetilde{x_{n}}+\widetilde{y_{n}}=2 x_{0}
$$

and

$$
\begin{aligned}
& \lim _{n \rightarrow \infty}\left\|\widetilde{x_{n}}\right\|_{\Phi, \Phi_{1}} \leq 1, \\
& \lim _{n \rightarrow \infty}\left\|\tilde{y_{n}}\right\|_{\Phi, \Phi_{1}} \leq 1 .
\end{aligned}
$$

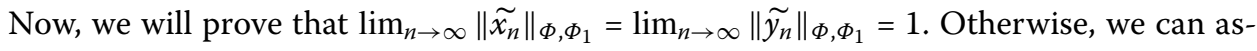
sume that $\lim _{n \rightarrow \infty}\left\|\tilde{x_{n}}\right\|_{\Phi, \Phi_{1}}<1$ and there exist $\delta>0, n_{0} \in N$ such that

$$
\begin{aligned}
\left\|\widetilde{x_{n}}\right\|_{\Phi, \Phi_{1}} & \leq 1-\delta, \\
\left\|\widetilde{y_{n}}\right\|_{\Phi, \Phi_{1}} & \leq 1+\frac{\delta}{2}
\end{aligned}
$$

for all $n \geq n_{0}$. Then

$$
1=\left\|x_{0}\right\|_{\Phi, \Phi_{1}}=\left\|\frac{\widetilde{x_{n}}+\widetilde{y_{n}}}{2}\right\|_{\Phi, \Phi_{1}} \leq \frac{1}{2}\left(1-\delta+1+\frac{\delta}{2}\right)<1
$$

a contradiction.

Thus

$$
\lim _{n \rightarrow \infty}\left\|\tilde{x_{n}}\right\|_{\Phi, \Phi_{1}}=\lim _{n \rightarrow \infty}\left\|\tilde{y_{n}}\right\|_{\Phi, \Phi_{1}}=1
$$

Since $\left\|\widetilde{x_{n}}-\tilde{y_{n}}\right\|_{\Phi, \Phi_{1}} \rightarrow 0$ if and only if $\left\|x_{n}-y_{n}\right\|_{\Phi, \Phi_{1}} \rightarrow 0(n \rightarrow \infty)$, we will use the sequences $\left\{\tilde{x_{n}}\right\}$ and $\left\{\tilde{y_{n}}\right\}$ instead of $\left\{x_{n}\right\}$ and $\left\{y_{n}\right\}$, respectively. Put $k_{n}^{\prime}=\frac{2 k_{n} k_{0}}{k_{n}+k_{0}}, h_{n}^{\prime}=\frac{2 h_{n} k_{0}}{h_{n}+k_{0}}$. Then $d=$ $\sup \left\{k_{n}^{\prime}, h_{n}^{\prime}\right\}<+\infty$.

Taking advantage of the forced convergence theorem and

$$
\begin{aligned}
\left\|\widetilde{x_{n}}\right\|_{\Phi, \Phi_{1}} & \leq \frac{1}{k_{n}^{\prime}}\left(1+\Phi\left(I_{\Phi_{1}}\left(k_{n}^{\prime} \widetilde{x_{n}}\right)\right)\right) \\
& \leq \frac{k_{n}+k_{0}}{2 k_{n} k_{0}}\left(1+\Phi\left(I_{\Phi_{1}}\left(\frac{k_{n} k_{0}}{k_{n}+k_{0}}\left(x_{n}+x_{0}\right)\right)\right)\right) \\
& \leq \frac{1}{2}\left(\frac{1}{k_{0}}+\frac{1}{k_{0}} \Phi\left(I_{\Phi_{1}}\left(k_{0} x_{0}\right)\right)+\frac{1}{k_{n}}+\frac{1}{k_{n}} \Phi\left(I_{\Phi_{1}}\left(k_{n} x_{n}\right)\right)\right) \\
& \leq \frac{1}{2}\left(\left\|x_{0}\right\|_{\Phi, \Phi_{1}}+\left\|x_{n}\right\|_{\Phi, \Phi_{1}}+\frac{1}{n}\right) \\
& \rightarrow 1 \quad(n \rightarrow \infty),
\end{aligned}
$$


we have

$$
\lim _{n \rightarrow \infty} \frac{1}{k_{n}^{\prime}}\left(1+\Phi\left(I_{\Phi_{1}}\left(k_{n}^{\prime} \tilde{x_{n}}\right)\right)\right)=1
$$

In the same way, we also have

$$
\lim _{n \rightarrow \infty} \frac{1}{h_{n}^{\prime}}\left(1+\Phi\left(I_{\Phi_{1}}\left(h_{n}^{\prime} \tilde{y_{n}}\right)\right)\right)=1
$$

Assume $k_{n}^{\prime} \rightarrow k, h_{n}^{\prime} \rightarrow h(n \rightarrow \infty)$. We will prove that $k, h \geq 1$. Since $\lim _{n \rightarrow \infty} \frac{1}{k_{n}^{\prime}}(1+$ $\left.\Phi\left(I_{\Phi_{1}}\left(k_{n}^{\prime} \tilde{x_{n}}\right)\right)\right)=1$, then $\lim _{n \rightarrow \infty} \Phi\left(I_{\Phi_{1}}\left(k_{n}^{\prime} \tilde{x_{n}}\right)\right)=k-1$. If $k<1$, then $\Phi\left(I_{\Phi_{1}}\left(k_{n}^{\prime} \tilde{x_{n}}\right)\right)<0$ as $n \rightarrow$ $\infty$, a contradiction. Therefore, $k \geq 1$. Similarly, $h \geq 1$.

Hence

$$
\frac{k}{k+h}, \frac{h}{k+h} \in\left[\frac{1}{1+d}, \frac{d}{1+d}\right]
$$

In order to finish the proof of the theorem, we divide the left proof of the theorem into three steps.

Step 1: We will show that $k_{0}=\frac{2 k h}{k+h} \in K\left(x_{0}\right)$. In fact

$$
\begin{aligned}
\left\|x_{0}\right\|_{\Phi, \Phi_{1}} & \leq \frac{k_{n}^{\prime}+h_{n}^{\prime}}{2 k_{n}^{\prime} h_{n}^{\prime}}\left(1+\Phi\left(I_{\Phi_{1}}\left(\frac{2 k_{n}^{\prime} h_{n}^{\prime}}{k_{n}^{\prime}+h_{n}^{\prime}} x_{0}\right)\right)\right) \\
& \leq \frac{k_{n}^{\prime}+h_{n}^{\prime}}{2 k_{n}^{\prime} h_{n}^{\prime}}\left(1+\Phi\left(I_{\Phi_{1}}\left(\frac{k_{n}^{\prime} h_{n}^{\prime}}{k_{n}^{\prime}+h_{n}^{\prime}}\left(\widetilde{x_{n}}+\widetilde{y_{n}}\right)\right)\right)\right) \\
& \left.\leq \frac{k_{n}^{\prime}+h_{n}^{\prime}}{2 k_{n}^{\prime} h_{n}^{\prime}}\left(1+\Phi\left(I_{\Phi_{1}}\left(\frac{h_{n}^{\prime}}{k_{n}^{\prime}+h_{n}^{\prime}} k_{n}^{\prime} \widetilde{x_{n}}+\frac{k_{n}^{\prime}}{k_{n}^{\prime}+h_{n}^{\prime}} h_{n}^{\prime} \tilde{y_{n}}\right)\right)\right)\right) \\
& \leq \frac{1}{2}\left(\frac{1}{k_{n}^{\prime}}\left(1+\Phi\left(I_{\Phi_{1}}\left(k_{n}^{\prime} \widetilde{x_{n}}\right)\right)\right)+\frac{1}{h_{n}^{\prime}}\left(1+\Phi\left(I_{\Phi_{1}}\left(h_{n}^{\prime} \tilde{y_{n}}\right)\right)\right)\right) \\
& \rightarrow 1 \quad(n \rightarrow \infty) .
\end{aligned}
$$

Since $\left\|x_{0}\right\|_{\Phi, \Phi_{1}}=1$, we get $\frac{2 k_{n}^{\prime} h_{n}^{\prime}}{k_{n}^{\prime} h_{n}^{\prime}} \rightarrow \frac{2 k h}{k+h}=k_{0} \in K\left(x_{0}\right)$.

Step 2: We will prove that $k_{n}^{\prime} \tilde{x_{n}}-k_{0} x_{0} \stackrel{\mu}{\rightarrow} 0(n \rightarrow \infty)$.

Firstly, we will show that

$$
k \tilde{x_{n}}-h \widetilde{y_{n}} \stackrel{\mu}{\rightarrow} 0 \quad(n \rightarrow \infty) .
$$

Otherwise, there exist $\sigma_{0}, \varepsilon_{0}>0$ such that

$$
\mu\left(\left\{t \in G:\left|k \tilde{x_{n}}(t)-h \tilde{y_{n}}(t)\right| \geq \sigma_{0}\right\}\right) \geq \varepsilon_{0} .
$$

Let

$$
\begin{aligned}
& D=\Phi_{1}^{-1}\left(\frac{3}{\varepsilon_{0}}\right), \\
& D_{1}=2 k D .
\end{aligned}
$$


Put $G_{n}=\left\{t \in G:\left|k \tilde{x_{n}}(t)\right| \leq D_{1},\left|h \tilde{y_{n}}(t)\right| \leq D_{1},\left|k \tilde{x_{n}}(t)-h \tilde{y_{n}}(t)\right| \geq \sigma_{0}\right\}$. We will show that

$$
\mu\left(G_{n}\right)>\frac{\varepsilon_{0}}{3} .
$$

Indeed, since $\lim _{n \rightarrow \infty}\left\|\widetilde{x_{n}}\right\|_{\Phi, \Phi_{1}}=1$, we may assume $\left\|\widetilde{x_{n}}\right\|_{\Phi} \leq\left\|\widetilde{x_{n}}\right\|_{\Phi, \Phi_{1}} \leq 2$. Then

$$
\begin{aligned}
1 & \geq I_{\Phi_{1}}\left(\frac{\widetilde{x_{n}}}{2}\right) \\
& \geq \int_{\left\{t \in G: \mid \widetilde{x_{n}}(t)\right.} \Phi_{1} \Phi_{1}\left(\frac{\widetilde{x_{n}}(t)}{2}\right) d t \\
& >\Phi_{1}(D) \mu\left(\left\{t \in G:\left|\frac{\widetilde{x_{n}}(t)}{2}\right|>D\right\}\right) \\
& =\frac{3}{\varepsilon_{0}} \mu\left(\left\{t \in G:\left|\frac{\widetilde{x_{n}}(t)}{2}\right|>D\right\}\right) .
\end{aligned}
$$

Hence

$$
\mu\left(\left\{t \in G:\left|\frac{\widetilde{x_{n}}(t)}{2}\right|>D\right\}\right)<\frac{\varepsilon_{0}}{3}
$$

Consequently,

$$
\mu\left(\left\{t \in G:\left|k \tilde{x_{n}}(t)\right|>D_{1}\right\}\right)<\frac{\varepsilon_{0}}{3} .
$$

Therefore,

$$
\begin{aligned}
\mu\left(G_{n}\right)= & \mu\left(\left\{t \in G:\left|k \tilde{x_{n}}(t)-h \tilde{y_{n}}(t)\right| \geq \sigma_{0}\right\}\right)-\mu\left(\left\{t \in G:\left|k \tilde{x_{n}}(t)\right|>D_{1}\right\}\right) \\
& -\mu\left(\left\{t \in G:\left|h \tilde{y_{n}}(t)\right|>D_{1}\right\}\right) \\
> & \varepsilon_{0}-\frac{\varepsilon_{0}}{3}-\frac{\varepsilon_{0}}{3} \\
= & \frac{\varepsilon_{0}}{3} .
\end{aligned}
$$

Let

$$
F=\left\{(x, y):|x| \leq D_{1},|y| \leq D_{1},|x-y| \geq \sigma_{0}, \frac{h}{k+h} x+\frac{k}{k+h} y \in S_{\Phi}\right\} .
$$

By virtue of the fact that $S_{\Phi_{1}}$ is a closed set, we know that $F$ is a bounded closed set and

$$
f(x, y)=\frac{\Phi_{1}\left(\frac{h}{k+h} x+\frac{k}{k+h} y\right)}{\frac{h}{k+h} \Phi_{1}(x)+\frac{k}{k+h} \Phi_{1}(y)}<1
$$

for every $(x, y) \in F$.

By $f(x, y)$ is continuous on $F$, there exists $\left(x_{0}, y_{0}\right) \in F$ such that $f(x, y) \leq f\left(x_{0}, y_{0}\right)$. We next will prove that $f\left(x_{0}, y_{0}\right)<1$. If $f\left(x_{0}, y_{0}\right)=1$, then $\frac{\Phi_{1}\left(\frac{h}{k+h} x_{0}+\frac{k}{k+h} y_{0}\right)}{\frac{h}{k+h} \Phi_{1}\left(x_{0}\right)+\frac{k}{k+h} \Phi_{1}\left(y_{0}\right)}=1$, this contradicts $\frac{h}{k+h} x_{0}+\frac{k}{k+h} y_{0} \in S_{\Phi_{1}}$. Put $f\left(x_{0}, y_{0}\right)=1-\delta$. For every $(x, y) \in F$, we have

$$
\Phi_{1}\left(\frac{h}{k+h} x+\frac{k}{k+h} y\right) \leq(1-\delta)\left(\frac{h}{k+h} \Phi_{1}(x)+\frac{k}{k+h} \Phi_{1}(y)\right) .
$$


By the definition of $k_{0}$ and $x_{0}$, we derive that

$$
\frac{h}{k+h} k \tilde{x_{n}}(t)+\frac{k}{k+h} h \tilde{y_{n}}(t)=\frac{2 k h}{k+h} x_{0}(t)=k_{0} x_{0}(t) \in S_{\Phi_{1}} .
$$

Since $k_{0} x_{0}(t) \in S_{\Phi_{1}}$, then $\left(k \widetilde{x_{n}}(t), h \tilde{y_{n}}(t)\right) \in F$, i.e., for $t \in G_{n}$ and

$$
\Phi_{1}\left(\frac{h}{k+h} k \tilde{x_{n}}(t)+\frac{k}{k+h} h \tilde{y_{n}}(t)\right) \leq(1-\delta)\left(\frac{h}{k+h} \Phi_{1}\left(k \tilde{x_{n}}(t)\right)+\frac{k}{k+h} \Phi_{1}\left(h \tilde{y_{n}}(t)\right)\right) .
$$

Hence

$$
\begin{aligned}
& \left\|\tilde{x_{n}}+\tilde{y_{n}}\right\|_{\Phi, \Phi_{1}} \\
& \leq \frac{k+h}{k h}\left(1+\Phi\left(I_{\Phi_{1}}\left(\frac{k h}{k+h}\left(\tilde{x_{n}}+\tilde{y_{n}}\right)\right)\right)\right. \\
& \leq \frac{k+h}{k h}+\frac{k+h}{k h} \Phi\left(\int_{G} \Phi_{1}\left(\frac{k h}{k+h}\left(\tilde{x_{n}}(t)+\tilde{y_{n}}(t)\right)\right) d t\right) \\
& \leq \frac{k+h}{k h}+\frac{k+h}{k h} \Phi\left((1-\delta) \int_{G_{n}}\left[\frac{h}{k+h} \Phi_{1}\left(k \tilde{x_{n}}(t)\right)+\frac{k}{k+h} \Phi_{1}\left(h \tilde{y_{n}}(t)\right)\right] d t\right) \\
& +\frac{k+h}{k h} \Phi\left(\int_{G \backslash G_{n}}\left[\frac{h}{k+h} \Phi_{1}\left(k \tilde{x_{n}}(t)\right)+\frac{k}{k+h} \Phi_{1}\left(h \tilde{y_{n}}(t)\right)\right] d t\right) \\
& \leq \frac{k+h}{k h}+\frac{k+h}{k h} \Phi\left(\int_{G}\left[\frac{h}{k+h} \Phi_{1}\left(k \tilde{x_{n}}(t)\right)+\frac{k}{k+h} \Phi_{1}\left(h \tilde{y_{n}}(t)\right)\right] d t\right) \\
& -\frac{k+h}{k h} \Phi\left(\delta \int_{G_{n}}\left[\frac{h}{k+h} \Phi_{1}\left(k \tilde{x_{n}}(t)\right)+\frac{k}{k+h} \Phi_{1}\left(h \tilde{y_{n}}(t)\right)\right] d t\right) \\
& \leq \frac{1}{k}\left(1+\Phi\left(I_{\Phi_{1}}\left(k \tilde{x_{n}}(t)\right)\right)+\frac{1}{h}\left(1+\Phi\left(I_{\Phi_{1}}\left(k \tilde{y_{n}}(t)\right)\right)\right.\right. \\
& -\frac{k+h}{k h} \Phi\left(\delta \int_{G_{n}}\left[\frac{h}{k+h} \Phi_{1}\left(k \tilde{x_{n}}(t)\right)+\frac{k}{k+h} \Phi_{1}\left(h \tilde{y_{n}}(t)\right)\right] d t\right) \text {. }
\end{aligned}
$$

Notice that

$$
I_{\Phi_{1}}\left(\left(k-k_{n}^{\prime}\right) \tilde{x_{n}}\right) \leq\left|k-k_{n}^{\prime}\right| I_{\Phi_{1}}\left(\tilde{x_{n}}\right) \rightarrow 0 \quad(n \rightarrow \infty) .
$$

By Lemma 2.11, we get

$$
I_{\Phi_{1}}\left(k \tilde{x_{n}}\right)-I_{\Phi_{1}}\left(k_{n}^{\prime} \tilde{x_{n}}\right)=I_{\Phi_{1}}\left(k_{n}^{\prime} \tilde{x_{n}}+\left(k-k_{n}^{\prime}\right) \tilde{x_{n}}\right)-I_{\Phi_{1}}\left(k_{n}^{\prime} \tilde{x_{n}}\right) \rightarrow 0 \quad(n \rightarrow \infty) .
$$

Thus

$$
\begin{aligned}
0 & \leq \frac{1}{k}\left(1+\Phi\left(I_{\Phi_{1}}\left(k \tilde{x_{n}}\right)\right)\right)-\left\|\tilde{x_{n}}\right\|_{\Phi, \Phi_{1}} \\
& =\frac{1}{k}\left(1+\Phi\left(I_{\Phi_{1}}\left(k \tilde{x_{n}}\right)\right)\right)-\frac{1}{k_{n}^{\prime}}\left(1+\Phi\left(I_{\Phi_{1}}\left(k_{n}^{\prime} \tilde{x_{n}}\right)\right)\right)+\frac{1}{n} \rightarrow 0 \quad(n \rightarrow \infty) .
\end{aligned}
$$

Similarly, $\frac{1}{h}\left(1+\Phi\left(I_{\Phi_{1}}\left(h \tilde{y_{n}}\right)\right)\right)-\left\|\tilde{y_{n}}\right\|_{\Phi, \Phi_{1}} \rightarrow 0$ as $n \rightarrow \infty$. Since $u>0, \Phi(u)>0$, and

$$
\left\|\tilde{x_{n}}+\widetilde{y_{n}}\right\|_{\Phi, \Phi_{1}} \leq 2-\frac{k+h}{k h}\left(\Phi\left(\frac{2 \delta}{1+d} \Phi_{1}\left(\frac{\delta_{0}}{2}\right) \frac{\varepsilon_{0}}{3}\right)\right) \quad(n \rightarrow \infty),
$$

we have $\lim _{n \rightarrow \infty}\left\|\widetilde{x_{n}}+\tilde{y_{n}}\right\|_{\Phi, \Phi_{1}}<2$. A contradiction. Hence $k \widetilde{x_{n}}-h \widetilde{y_{n}} \stackrel{\mu}{\rightarrow} 0$. 
Thanks to the $\Phi$-Amemiya norm being equivalent with the Luxemburg norm, their weak topology and weak star topology are all equivalent. So $L_{\Phi, \Phi_{1}}$ is $w^{*}$ compact. Take $\left\{x_{n}^{\prime \prime}\right\} \subset\left\{\widetilde{x_{n}}\right\},\left\{y_{n}^{\prime \prime}\right\} \subset\left\{\widetilde{y_{n}}\right\}$ such that $x_{n}^{\prime \prime} \stackrel{w^{*}}{\rightarrow} x^{\prime}$ and $y_{n}^{\prime \prime} \stackrel{w^{*}}{\rightarrow} y^{\prime}$. We get $x^{\prime}+y^{\prime}=2 x_{0}$.

Since $\Phi_{1} \in \triangle_{2}$, we have

$$
\|x\|_{\Phi, \Phi_{1}}=\sup \left\{\int_{G} x(t) y(t) d t: y \in B\left(L_{\Phi, \Phi_{1}}^{*}\right)\right\},
$$

where $L_{\Phi, \Phi_{1}}^{*}$ is the dual space of $L_{\Phi, \Phi_{1}}$.

So

$$
\|x\|_{\Phi, \Phi_{1}}=\sup \left\{\int_{G} x(t) y(t) d t: y \in B\left(E_{\Phi, \Phi_{1}}^{*}\right)\right\} .
$$

Since $\left\|2 x_{0}\right\|_{\Phi, \Phi_{1}}=2$, then

$$
\left\|2 x_{0}\right\|_{\Phi, \Phi_{1}} \leq\left\|x^{\prime}\right\|_{\Phi, \Phi_{1}}+\left\|y^{\prime}\right\|_{\Phi, \Phi_{1}} \leq \underline{\lim }_{n \rightarrow \infty}\left\|\widetilde{x_{n}}\right\|_{\Phi, \Phi_{1}}+\underline{\lim }_{n \rightarrow \infty}\left\|\widetilde{y_{n}}\right\|_{\Phi, \Phi_{1}}=2 .
$$

This shows

$$
\left\|x^{\prime}\right\|_{\Phi, \Phi_{1}}=\left\|y^{\prime}\right\|_{\Phi, \Phi_{1}}=1
$$

Hence, there exist $k, h>1$ such that

$$
\begin{aligned}
& 1=\left\|x^{\prime}\right\|_{\Phi, \Phi_{1}}=\frac{1}{k}\left(1+\Phi\left(I_{\Phi_{1}}\left(k x^{\prime}\right)\right)\right), \\
& 1=\left\|y^{\prime}\right\|_{\Phi, \Phi_{1}}=\frac{1}{h}\left(1+\Phi\left(I_{\Phi_{1}}\left(h y^{\prime}\right)\right)\right) .
\end{aligned}
$$

Since $\left\|x^{\prime}\right\|_{\Phi, \Phi_{1}}+\left\|y^{\prime}\right\|_{\Phi, \Phi_{1}}=2$, then

$$
\begin{aligned}
\left\|x^{\prime}\right\|_{\Phi, \Phi_{1}}+\left\|y^{\prime}\right\|_{\Phi, \Phi_{1}} & =\frac{1}{k}\left(1+\Phi\left(I_{\Phi_{1}}\left(k x^{\prime}\right)\right)\right)+\frac{1}{h}\left(1+\Phi\left(I_{\Phi_{1}}\left(h y^{\prime}\right)\right)\right) \\
& =\frac{k+h}{k h}\left[1+\frac{h}{k+h} \Phi\left(I_{\Phi_{1}}\left(k x^{\prime}\right)\right)+\frac{k}{k+h} \Phi\left(I_{\Phi_{1}}\left(h y^{\prime}\right)\right)\right] \\
& \geq \frac{k+h}{k h}\left[1+\Phi\left(\frac{h}{k+h}\left(I_{\Phi_{1}}\left(k x^{\prime}\right)\right)+\frac{k}{k+h}\left(I_{\Phi_{1}}\left(h y^{\prime}\right)\right)\right)\right] \\
& \geq \frac{k+h}{k h}\left[1+\Phi\left(I_{\Phi_{1}}\left(\frac{k h}{k+h} x^{\prime}+\frac{k h}{k+h} y^{\prime}\right)\right)\right] \\
& =2 \cdot \frac{1}{k_{0}}\left(1+\Phi\left(I_{\Phi_{1}}\left(k_{0} x_{0}\right)\right)\right) \\
& =2,
\end{aligned}
$$

and

$$
\left\|x_{0}\right\|_{\Phi, \Phi_{1}}=1=\frac{1}{k_{0}}\left(1+\Phi\left(I_{\Phi_{1}}\left(k_{0} x_{0}\right)\right)\right) .
$$

Hence $x^{\prime}=y^{\prime}=x_{0}$. Combining this with $k x_{n}^{\prime \prime}-h y_{n}^{\prime \prime} \stackrel{\mu}{\rightarrow} 0$, we can prove that $k x_{n}^{\prime \prime}-h y_{n}^{\prime \prime} \stackrel{w^{*}}{\rightarrow} 0$. Since $v(t) \in E_{\Psi}$, then for any $\varepsilon>0$ there exists $\delta>0$ such that $\left\|\nu \chi_{G_{0}}\right\|_{\Phi}<\varepsilon$, whence $\mu\left(G_{0}\right)<$ 
$\delta$. Using the EropoB theorem, there exists $G_{0} \subset G, \mu\left(G_{0}\right)<\delta$ such that $k x_{n}^{\prime \prime}-h y_{n}^{\prime \prime} \stackrel{\mu}{\rightarrow} 0$ for $t \in G \backslash G_{0}$.

Put $\left\|x_{n}^{\prime \prime}\right\|_{\Phi}^{o}=1$ and $\left\|y_{n}^{\prime \prime}\right\|_{\Phi}^{o}=1$. We have

$$
\begin{aligned}
\int_{G}\left(k x_{n}^{\prime \prime}-h y_{n}^{\prime \prime}\right) v(t) d t & =\int_{G \backslash G_{0}}\left(k x_{n}^{\prime \prime}-h y_{n}^{\prime \prime}\right) v(t) d t+\int_{G_{0}}\left(k x_{n}^{\prime \prime}-h y_{n}^{\prime \prime}\right) v(t) d t \\
& \leq M \cdot \mu\left(G \backslash G_{0}\right) \cdot \varepsilon+k\left\|x_{n}^{\prime \prime}\right\|_{\Phi}^{o}\|v(t)\|_{\Phi}+h\left\|y_{n}^{\prime \prime}\right\|_{\Phi}^{o}\|v(t)\|_{\Phi} \\
& \leq M \cdot \mu\left(G \backslash G_{0}\right) \cdot \varepsilon+k \cdot \varepsilon+h \cdot \varepsilon .
\end{aligned}
$$

By the arbitrariness of $\varepsilon$, we have $\int_{G}\left(k x_{n}^{\prime \prime}-h y_{n}^{\prime \prime}\right) v(t) d t<\varepsilon$. Thus $k x_{n}^{\prime \prime}-h y_{n}^{\prime \prime} \stackrel{w^{*}}{\rightarrow} 0$. Since $x_{n}^{\prime \prime}-y_{n}^{\prime \prime} \stackrel{w^{*}}{\rightarrow} 0$, then $k=h$. Thus $\widetilde{x_{n}}-\widetilde{y_{n}} \stackrel{\mu}{\rightarrow} 0$ as $n \rightarrow \infty$. Therefore

$$
k_{n}^{\prime} \tilde{x_{n}}-k_{0} x_{0} \stackrel{\mu}{\rightarrow} 0 \quad(n \rightarrow \infty)
$$

Step 3: We will prove that $I_{\Phi_{1}}\left(k_{n} x_{n}\right) \rightarrow I_{\Phi_{1}}\left(k_{0} x_{0}\right)$. In fact

$$
\begin{aligned}
& \Phi\left(I_{\Phi_{1}}\left(k_{0} x_{0}\right)\right)=k_{0}-1, \\
& \Phi\left(I_{\Phi_{1}}\left(k_{n}^{\prime} \widetilde{x_{n}}\right)\right) \rightarrow k \quad(n \rightarrow \infty) .
\end{aligned}
$$

We deduce that $\Phi\left(I_{\Phi_{1}}\left(k_{n}^{\prime} \tilde{x_{n}}\right)\right) \rightarrow \Phi\left(I_{\Phi_{1}}\left(k_{0} x_{0}\right)\right)(n \rightarrow \infty)$. Using $u>0, \Phi(u)>0$ and $\Phi(u)$ is strictly increasing, we get

$$
\left.I_{\Phi_{1}}\left(k_{n}^{\prime} \tilde{x_{n}}\right)\right) \rightarrow I_{\Phi_{1}}\left(k_{0} x_{0}\right) \quad(n \rightarrow \infty) .
$$

By Lemma 2.12, we have

$$
\left\|k_{n}^{\prime} \tilde{x_{n}}-k_{0} x_{0}\right\|_{\Phi, \Phi_{1}} \rightarrow 0
$$

Corollary 3.2 Let $\Phi$ be an Orlicz function. Then $L_{\Phi, \Phi_{1}}$ is midpoint local uniform rotundity if and only if $\Phi_{1} \in \Delta_{2}$ and $L_{\Phi, \Phi_{1}}$ is strictly convex.

\section{Acknowledgements}

The authors are extremely grateful to the reviewers for their valuable suggestions and their crucial role in leading to a better presentation of this manuscript.

\section{Funding}

This research is funded by the National Nature Science Foundation of China, under Grant 11871181.

\section{Availability of data and materials}

Not applicable.

\section{Competing interests}

The authors declare that they have no competing interests.

\section{Authors' contributions}

The authors declare that the study was realized in collaboration with the same responsibility. All authors read and approved the final manuscript. All authors contributed equally to writing of this paper.

\section{Authors' information}

Department of Mathematics, Harbin University of Science and Technology, No.52 Xuefu Road, Nangang District, Harbin City, Heilongjiang Province, China. 


\section{Publisher's Note}

Springer Nature remains neutral with regard to jurisdictional claims in published maps and institutional affiliations.

Received: 2 February 2020 Accepted: 29 July 2020 Published online: 06 August 2020

\section{References}

1. Phelps, R.: Dentability and extreme points in Banach spaces. J. Funct. Anal. 17(1), 78-90 (1974)

2. Rao, T.: Denting and strongly extreme points in the unit ball of spaces of operators. Proc. Indian Acad. Sci. Math. Sci. 109(1), 75-85 (1999)

3. Hudzik, H., Kurc, W., Wisła, M.: Strongly extreme points in Orlicz function spaces. J. Math. Anal. Appl. 189(3), 651-670 (1995)

4. Tang, X., Lin, Y:: A strong extreme point in Musielak-Orlicz spaces equipped with Orlicz norm. J. Guangdong Univ. Petrochem. Technol. 23(01), 58-61 (2013)

5. Chen, L., Cui, Y., Hudzik, H.: Criteria for complex strongly extreme points of Musielak-Orlicz function spaces. Nonlinear Anal., Theory Methods Appl. 70(6), 2270-2276 (2009)

6. Kunen, K., Rosenthal, H.: Martingale proofs of some geometrical results in Banach space theory. Pac. J. Math. 100(1), 153-175 (1982)

7. Musielak, J.: Orlicz spaces and modular spaces. Lect. Notes Math. 1034(4), 1-216 (1983)

8. Rao, M.M.: Linear functionals on Orlicz spaces: general theory. Pac. J. Math. 25(3), 553-585 (1968)

9. Shang, S., Cui, Y., Fu, Y.: Extreme points and rotundity in Musielak-Orlicz-Bochner function spaces endowed with Orlicz norm. Abstr. Appl. Anal. 2010(10), 1 (2010)

10. Cui, Y., Hudzik, H., Li, J., et al.: Strongly extreme points in Orlicz spaces equipped with the p-Amemiya norm. Nonlinear Anal. 71(12), 6343-6364 (2009)

11. Wisła, M.: Orlicz spaces equipped with s-norms. J. Math. Anal. Appl. 483(2), 1-30 (2019)

12. Cui, Y., Hudzik, H., Płuciennik, R.: Extreme points and strongly extreme points in Orlicz spaces equipped with the Orlicz norm. Z. Anal. Anwend. 22(4), 789-817 (2003)

13. Turett, B.: Rotundity of Orlicz spaces. Indag. Math. 79(5), 462-469 (1976)

14. Hudzik, H., Kamińska, A., Mastyło, M.: Monotonicity and rotundity properties in Banach lattices. Rocky Mt. J. Math. 30(3), 933-950 (2000)

15. Hudzik, H.: Strongly extreme points in Köthe-Bochner spaces. Rocky Mt. J. Math. 23(3), 899-909 (1993)

16. Storozhuk, K.V.: Strongly normal cones and the midpoint locally uniform rotundity. Positivity 17(3), 935-940 (2013)

17. Bo, L., Cui, Y., Foralewski, P., Hudzik, H.: Local uniform rotundity and weak local uniform rotundity of Musielak-Orlicz sequence spaces endowed with the Orlicz norm. Nonlinear Anal. 69(5-6), 1559-1569 (2008)

18. Fang, L., Wang, T., Hudzik, H.: Uniform Gateaux differentiability and weak uniform rotundity of Musielak-Orlicz function spaces. Nonlinear Anal., Theory Methods Appl. 56(8), 1133-1149 (2004)

19. Chen, S.: Geometry of Orlicz Spaces. Dissertations Math., Warzawa (1996)

\section{Submit your manuscript to a SpringerOpen ${ }^{\circ}$ journal and benefit from:}

- Convenient online submission

- Rigorous peer review

- Open access: articles freely available online

- High visibility within the field

- Retaining the copyright to your article

Submit your next manuscript at $\boldsymbol{\nabla}$ springeropen.com 